\title{
PULMONARY MUCOEPIDERMOID CARCINOMA: DIAGNOSIS AND TREATMENT
}

\section{Shafaq Maqsood, Usman Ahmad, Umm-e-Kulsoom}

Department of Medical Oncology, Shaukat Khanum Memorial Cancer Hospital and Research Centre, Lahore, Pakistan

Received: 18 October 2017 / Accepted: 19 December 2017

\begin{abstract}
Pulmonary mucoepidermoid carcinoma (MEC) is a rare malignant neoplasm with the clinical picture mimicking infectious aetiologies in most of the patients. Hence, this rare entity poses a great challenge to the pathologist in terms of diagnosis and to the oncologist in terms of treatment. This case report aims to look at the clinicopathological features of pulmonary MEC, the role of immunohistochemical analysis in diagnosis and choice of chemotherapeutic agent. The objective of reporting this case on MEC is not only the rare frequency of this carcinoma but also to highlight the importance of adequate immunohistochemical analysis in establishing the diagnosis.
\end{abstract}

Key words: Lung cancer, mucoepidermoid carcinoma, chemotherapy

\section{Introduction}

Mucoepidermoid carcinoma (MEC) of the lung is a rare pulmonary cancer that accounts for $0.1-0.2 \%$ of lung tumours. ${ }^{[1-3]}$ Main presenting features are of cough, fever and haemoptysis. ${ }^{[3]}$ Histological and immunohistochemical analysis remain the mainstay in establishing diagnosis with radiology providing little additional help. ${ }^{[4]}$

We present a case report on a 25 -year-old female whose clinical presentation was with cough and haemoptysis. Initially, she was diagnosed with poorly differentiated squamous cell carcinoma and underwent pneumonectomy. Histological review of resected specimen showed MEC $4.3 \mathrm{~cm}$, high grade and with a pathological stage of pT2aN2. Immunohistochemical analysis showed $\mathrm{p} 63$ positive, thyroid tissue for 1 (TTF1) negative and periodic acid-Schiff-diastase (PASD)/ mucicarmine positive for mucin and was helpful in establishing the diagnosis.

\section{Case Report}

Our patient, a 25-year-old female with no comorbidities and 5 months gravid, presented to pulmonology clinic

Correspondence: Dr. Shafaq Maqsood, Department of Medical Oncology, Shaukat Khanum Cancer Hospital and Research Center Lahore, Pakistan.

Email: drshafaq@live.com with a history of cough for 3 years and haemoptysis for 1 month. Computed tomography (CT) scan from an outside facility which showed the right lower lobe mass and appearances suggestive of a T2, N2 and Mx primary right lower lobe lung tumour.

The patient underwent tumour biopsy at our hospital which showed poorly differentiated squamous cell carcinoma. Immunohistochemical analysis showed positive $\mathrm{p} 40$ and negative TTF. Keratinization was absent. After discussion in multidisciplinary team conference, pneumonectomy was planned.

Histopathology review showed lung parenchyma showing an invasive tumour composed of solid nests and sheets. The tumour showed two cell populations, predominantly comprising epidermoid cells and intermixed mucin filled cells with the presence of moderate to marked atypia, nuclear pleomorphism and brisk mitotic activity [Figure 1].

A diagnosis of MEC was made which was high grade $4.3 \mathrm{~cm}$ and with lymphovascular invasion. Four of nine regional nodes were positive including ipsilateral mediastinal/subcarinal node making it a stage of pT2aN2. Immunohistochemical analysis showed positive $\mathrm{p} 63$ and $\mathrm{PASD} /$ mucicarmine positive for mucin, whereas TTF1 was negative. The previous biopsy was also reviewed and the final diagnosis of MEC was made on the basis of 


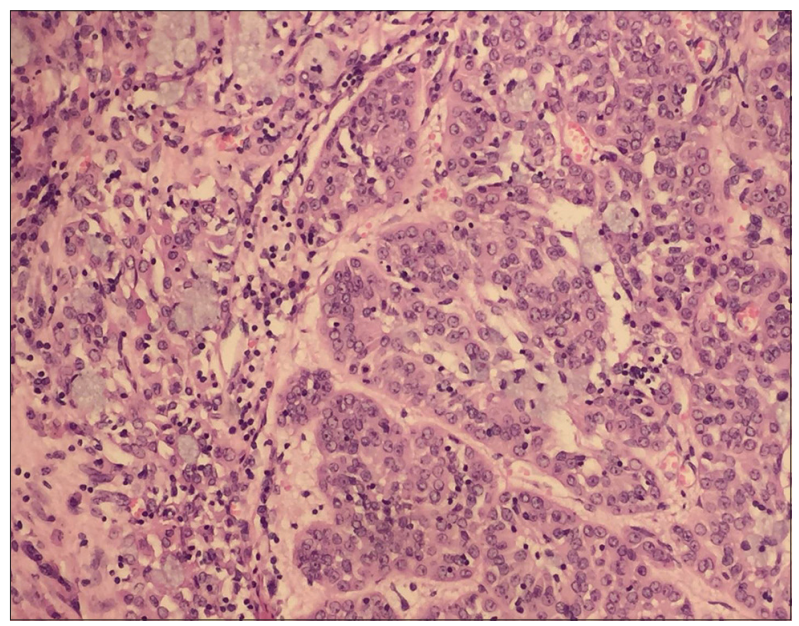

Figure 1: Lung parenchyma showing invasive tumour with two cell populations, predominantly epidermoid and mucinfilled cells

two cell populations, the absence of keratinization and a negative TTF1.

The patient was then seen in medical oncology clinic, chemotherapy was planned, but since the patient was pregnant, she was first referred to obstetrics for induction of labour. She came for chemotherapy after delivery of her child. A baseline CT scan was done and chemotherapy carboplatin AUC-5 and paclitaxel $175 \mathrm{mg} / \mathrm{m}^{2}$ every 3 weeks were started. There was an interval of 4.5 months between surgery and first chemotherapy cycle. Our patient received 6 cycles of carboplatin and paclitaxel without any significant toxicity. She remained on active surveillance and disease free for up to a year. Her disease relapsed at multiple sites, and unfortunately, she could not be given second-line chemotherapy in view of her florid disease and poor performance score.

\section{Discussion}

MEC is defined by the World Health Organisation as a tumour comprising mucus secreting, squamous and intermediate cells..$^{[1,4]}$ More frequently, it is found in the parotid and the submandibular salivary glands. ${ }^{[4]}$ MEC equally affects males and females both with the median age of presentation at 40 years; however, the range is wide from 3 to 78 years. ${ }^{[4,5]}$

In general, it involves the proximal bronchi, and hence, the patient presents typically with symptoms suggestive of bronchial obstruction such as cough, haemoptysis, wheezing, fever and post-obstructive pneumonia. ${ }^{[3,4]}$

Chest radiographs may show distal atelectasis or pneumonia and rarely help in diagnosis. CT scan generally shows non-spherical, smooth polypoidal mass. ${ }^{[5]}$

Histologically, the tumour can be classified as low grade or high grade. ${ }^{[6]}$ Low grade mostly has cystic components with mild atypia. High-grade tumours predominantly show squamoid and intermediate cell with a small component of mucin-secreting cells with high mitotic rate ${ }^{[7]}$ Making a diagnosis of high-grade MEC before surgery is difficult. It is the histological findings of the presence of three components, mucin secreting, squamous and intermediate that help establish the diagnosis of MEC. ${ }^{[4]}$ To distinguish MEC from adenosquamous carcinoma is not easy. The absence of keratinization and TTF1 negativity is suggestive of high-grade MEC. ${ }^{[8]}$

Surgical resection remains the standard treatment for pulmonary MEC. Video-assisted thoracoscopic surgery is becoming more common operative approach. ${ }^{[3]}$ In low-grade tumours, adjuvant therapy is not indicated. ${ }^{[5]}$

Prognosis of low-grade MEC is excellent with 5-year survival of $95 \%$. In contrast, high-grade MEC carries a poor prognosis with most of the patients succumbing to disease. ${ }^{[3,6]}$ Lymph node metastasis is the most important prognostic factor in pulmonary MEC and imparts a dismal outcome. Therefore, surgery alone does not seem to be adequate for such patients. ${ }^{[8]}$

The role of adjuvant chemotherapy and targeted agents has only been studied in case reports. Epithelial growth receptor (EGFR) mutation is found in $40 \%$ of cases of pulmonary MEC and gefitinib has shown effectiveness in such cases as reported ${ }^{[9]}$ However, there have also been reports of response to tyrosine kinase inhibitors (TKIs) in patients with no EGFR mutation which warrants further studies. ${ }^{[3,9]}$

\section{Conclusion}

Rare tumours pose a challenge to the pathologist and oncologist both in terms of diagnosis and treatment, respectively. MEC of the lung is one such entity. Literature review is available mainly in the form of case reports, and 
hence, there are no established chemotherapy protocols. The significance of this case report is to highlight the importance of histological and immunohistochemical analysis in the diagnosis of this infrequent tumour. One important aspect that was investigated was the use of carboplatin and paclitaxel as chemotherapeutic agents and assessment of disease response.

Needless to say, more studies are required to define the optimal treatment regimens for initial presentations as well as relapsed/refractory disease.

\section{Established facts}

- $\quad$ Rare tumour with clinical presentation mimicking infectious aetiology.

- Pathology and immunohistochemical analysis are essential for diagnosis with radiology having a limited role.

- Surgery as the mainstay of treatment in lowgrade MEC.

\section{Novel insights}

Role of chemotherapy in high-grade pulmonary MEC. In areas like ours, the resources are limited and a number of patients have financial restraints which of course mean that testing for EGFR mutations, and consequently, the use of TKIs is not really a practical option. Therefore, we have to rely on chemotherapy in adjuvant settings in case of high-grade tumours. The only literature on the choice of chemotherapeutic agents in pulmonary MEC is in the form of case reports and series. In the case of our patient with high-grade MEC, we used carboplatin and paclitaxel which provided a disease-free survival of 1 year.

\section{Conflict of Interest}

The authors declare that they have no conflict of interest.

\section{References}

1. Shen C, Che G. Clinicopathological analysis of pulmonary mucoepidermoid carcinoma. World J Surg Oncol 2014;12:33.

2. Kitada M, Matsuda Y, Sato K, et al. Mucoepidermoid carcinoma of the lung: A case report. J Cardiothorac Surg 2011;6:132.

3. Alsidawi S, Morris JC, Wikenheiser-Brokamp KA, et al. Mucoepidermoid carcinoma of the lung: A case report and literature review. Case Rep Oncol Med 2013;2013:625243.

4. Liu X, Adams AL. Mucoepidermoid carcinoma of the bronchus: A review. Arch Pathol Lab Med 2007;131:1400-4.

5. Chopra A, Shim C, Sharma N, et al. Primary salivary type lung tumor: Mucoepidermoid carcinoma. Respir Med Case Rep 2013;9:18-20.

6. Kuzucuoglu M, Karamustafaoglu YA, Cicin I, et al. A rarely seen mucoepidermoid carcinoma of the left main bronchus. J Cancer Res Ther 2014;10:384-6.

7. Belgod SR, Reddy RH, KumarSP. Mucoepidermoid carcinoma of the lung: A rare entity. Oxf Med Case Reports 2015; 2015:203-5.

8. Xi JJ, Jiang W, Lu SH, et al. Primary pulmonary mucoepidermoid carcinoma: An analysis of 21 cases. World J Surg Oncol 2012;10:232.

9. Han SW, Kim HP, Jeon YK, et al. Mucoepidermoid carcinoma of lung: Potential target of EGFR-directed treatment. Lung Cancer 2008;61:30-4. 